\title{
Differential Phase Contrast Scanning Transmission Electron Microscopy at Atomic Resolution
}

N. Shibata ${ }^{1,2}$

1. Institute of Engineering Innovation, The University of Tokyo, Tokyo 113-8656 Japan

2. Nanostructures Research Laboratory, Japan Fine Ceramic Center, Nagoya 456-8587, Japan

* Corresponding author: shibata@ sigma.t.u-tokyo.ac.jp

Understanding the atomic-scale structures of surfaces and interfaces is essential to control the functional properties of many materials and devices. Recent advances in aberration-corrected scanning transmission electron microscopy (STEM) have made possible to directly characterize localized structures in materials, especially at interfaces, in both atomic structures and chemistry. In STEM, a finely focused electron probe is scanned across the specimen and the transmitted and/or scattered electrons from a localized volume of the material are detected by the post-specimen detector(s). By controlling the detector geometry, we gain flexibility in determining the contrast characteristics of the STEM images and the formation mechanisms involved.

By elaborating special detector geometries, we can image local electromagnetic fields inside materials through differential phase contrast (DPC) imaging techniques [1,2]. We have been continuously developing very fast area detectors that are capable of atomic-resolution STEM imaging. These area detectors can obtain 16 simultaneous atomic-resolution STEM images which are sensitive to the spatial distribution of scattered electrons on the detector plane [3]. By applying these area detectors, atomicresolution DPC STEM imaging has been realized [4]. Figure 1 shows simultaneous (a) annular dark field (ADF) image, (b) electric field vector color map and (c) electric field strength map of $\mathrm{SrTiO}_{3}$ crystal observed along [001] direction, constructed from quantitative DPC STEM images [5,6]. Comparing with the simultaneous ADF image, disks of rotating color contrast are seen at each atomic column position in the electric field vector map. The direction of rotating color contrast is the same in all the atomic columns irrespective of the atomic species, visualizing that the (projected) atomic electric field points outward from the center of the atomic columns. Thus, we can now directly visualize electric field distribution within single atoms: the electric field between positive atomic nucleus and negative electron clouds. Converting such local electric field into total charge density, we can map total charge density distribution inside atoms as shown in Figure 2 [7]. We are able to map the spatial distribution of the electron cloud within individual atomic columns. We also found that DPC STEM imaging is very powerful to directly characterize many interesting internal electromagnetic structures in devices and materials which cannot be observed by normal STEM imaging techniques using annular type detectors. In this presentation, current status of our atomic-resolution DPC STEM developments and future directions will be discussed.

\section{References:}

[1] N.H. Dekkers and H. de Lang, Optik, 41, 452 (1974).

[2] N. Shibata et al., Acc. Chem. Res., 50, 1502-1512 (2017).

[3] N. Shibata et al., J. Electron Microscopy, 59, 473-479 (2010).

[4] N. Shibata et al., Nature Phys., 8, 611-615 (2012). 
[5] N. Shibata et al., Nature Comm. 8, 15631 (2017).

[6] T. Seki et al., Ultramicroscopy, 182, 258-263 (2017).

[7] G. Sánchez-Santolino et al., ACS Nano, 12, 8875 (2018).

[8] The author thank all the collaborators of this research, especially Drs. S.D. Findlay, Y. Kohno, T. Seki, R. Ishikawa, G. Sánchez-Santolino and Y. Ikuhara for their contribution to the works shown in this presentation. The author acknowledges support from the SENTAN, JST and the JSPS KAKENHI Grant number JP17H01316.

(a)

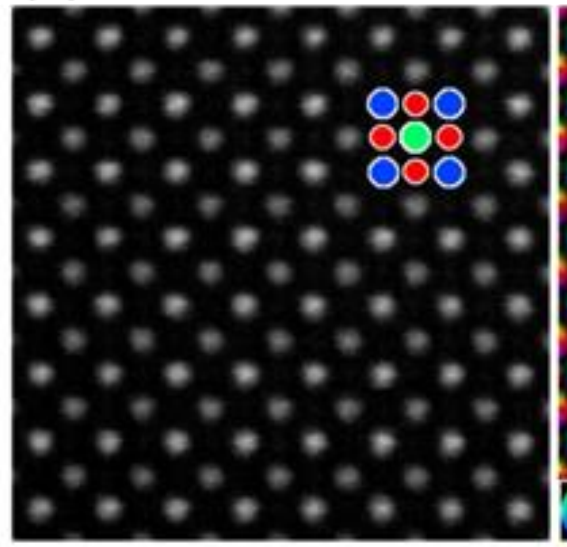

(b)

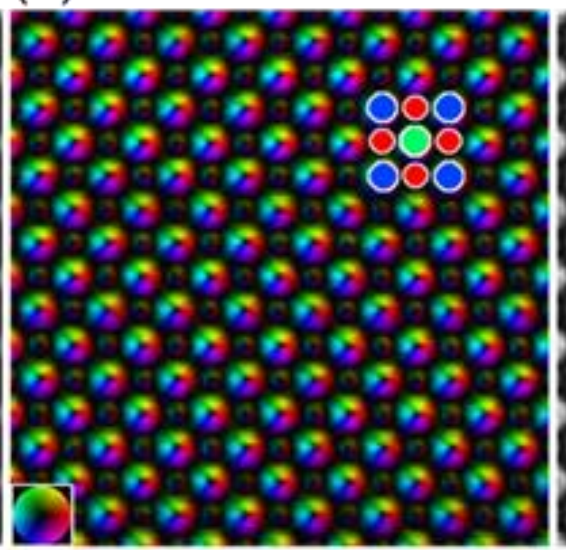

(c)

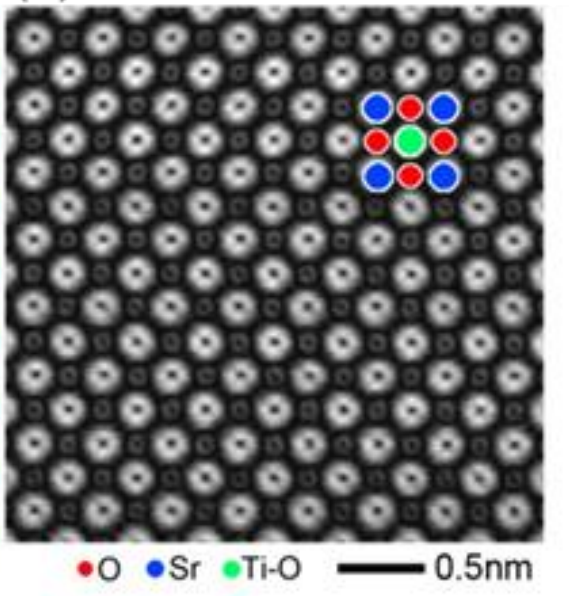

Figure 1. Atomic resolution DPC STEM images of $\mathrm{SrTiO}_{3}$ observed from [001] direction [5]. (a) ADF image. (b) Projected electric field vector color map and (c) electric field strength map constructed from the segmented detector DPC images. The inset color wheel indicates how color and shade denote the electric field orientation and strength in the vector color map.

(a)

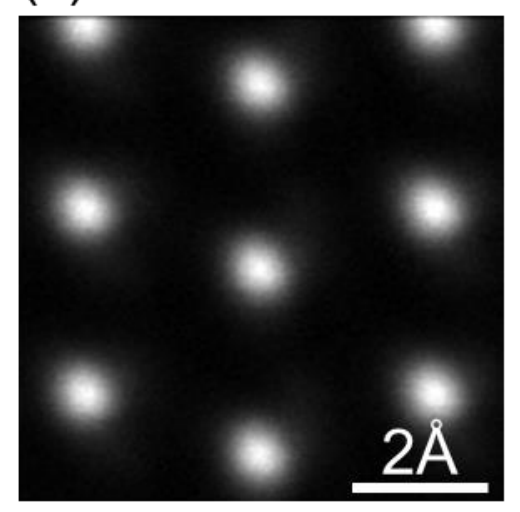

(b)

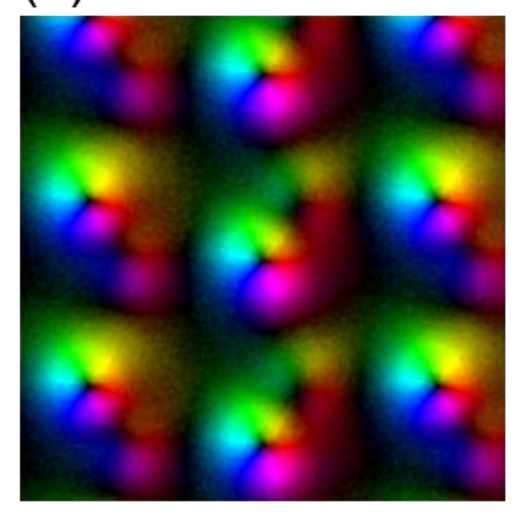

(c)

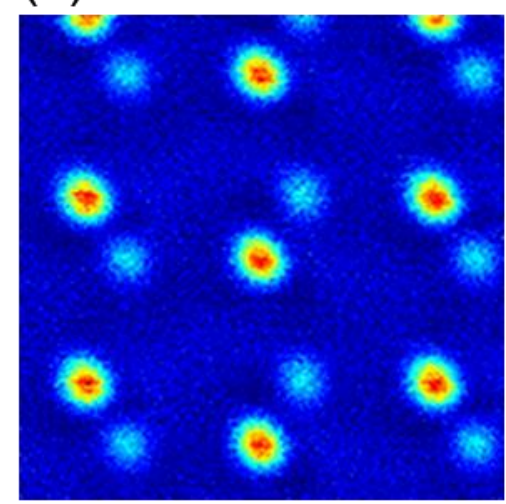

$-2 \mathrm{e} \AA^{-2}$

Figure 2. Quantitative atomic-scale mapping of the projected total charge density for GaN observed along [11-20] direction [7]. (a) ADF image. (b) Projected electric field. (c) Projected total charge density map calculated from (b). 